\title{
THERAPY
}

\section{Real-world trial of treat-to-target-less drop-out, shorter time to improvement}

Treat-to-target approaches, known to be superior to routine care in patients with early rheumatoid arthritis (RA), are also better in those with established disease, report Janet Pope and colleagues in Arthritis Care \& Research. "The unexpected finding was that there were far fewer drop outs in those who had treatment to a target," states Pope.

The 18-month real-world trial involved 308 patients with established RA for whom a decision to initiate therapy with adalimumab had been made independently of the study. "We did cluster randomization," explains Pope, "as it would be difficult for a rheumatologist to treat one person to a target and not another patient." Two different targets28 -joint disease activity score (DAS28) $<2.6(n=100)$ and swollen-joint count (SJC) of $0(n=99)$ - were compared with routine care $(n=109)$. Decisions regarding concomitant medication, routine care, and timing of appointments were left to physician judgement. Change in DAS28 between baseline and 18 months was the primary outcome measure; secondary measures included SJC and achievement of DAS28 $<2.6$ and EULAR responses.

In all three groups, 6-month improvements in DAS28 were significant, comparable, and maintained to 18 months. Between-group differences were nonsignificant for most secondary outcomes. Patients in both target groups, however, had significantly shorter time to achieving a good/moderate EULAR response in comparison with routine care. Furthermore, patient satisfaction increased significantly more in the DAS28 group than the other groups. The greatest difference was in retention: $34.4 \%$ of patients in the routine care group, compared with $27.0 \%$ in the DAS 28 group and $22.2 \%$ in the SJC group were discontinued by 18 months.

"Patients possibly achieve a target more quickly and they continue to use

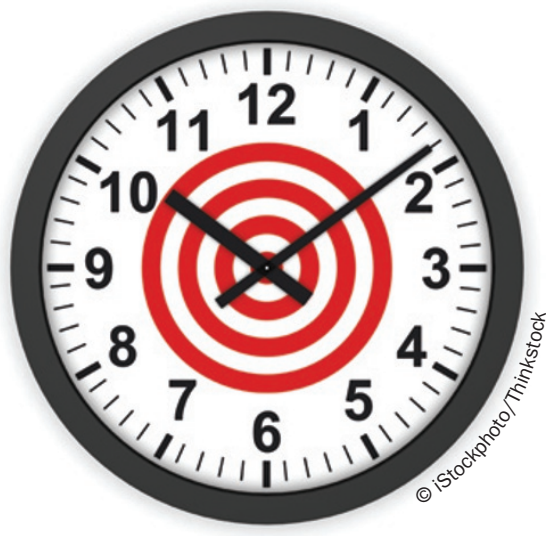

their medication - so their treatment is optimized," says Pope. "This is important as the drop out with TNF inhibitors over the first 2 years is very high." Noting that the findings show real-world trials to be capable of answering scientific questions through usual care she concludes: "maybe we should be doing this more often."

Emma Leah

Original article Pope, J. et al. Treating to a target in established active RA receiving a TNF inhibitor. Results from the optimization of adalimumab randomized trial. Arthritis Care Res. doi:10.1002/acr.22010 